BMJ Open

Diabetes

Research

\& Care

\section{Influence of guideline adherence and parameter control on the clinical outcomes in patients with diabetic nephropathy}

Jingru Lu, ${ }^{1,2}$ Wei Zhao, ${ }^{3}$ Tingyu Chen, ${ }^{2}$ Zhuoyang $\mathrm{Xu},{ }^{3}$ Xingzhi Sun, ${ }^{3}$ Honglang Xie, ${ }^{2}$ Yu An, ${ }^{2}$ Caihong Zeng, ${ }^{2}$ Gang Hu, ${ }^{3}$ Guotong Xie, ${ }^{3}$ Zhihong Liu (1) ${ }^{1,2}$

To cite: Lu J, Zhao W, Chen T, et al. Influence of guideline adherence and parameter control on the clinical outcomes in patients with diabetic nephropathy. BMJ Open Diab Res Care 2020;8:e001166. doi:10.1136/ bmjdrc-2019-001166

- Additional material is published online only. To view please visit the journal online (http://dx.doi.org/10.1136/ bmjdrc-2019-001166).

JL and WZ contributed equally.

Received 29 December 2019 Revised 27 March 2020 Accepted 30 May 2020

\section{Check for updates}

\section{(C) Author(s) (or their} employer(s)) 2020. Re-use permitted under CC BY-NC. No commercial re-use. See rights and permissions. Published by BMJ.

${ }^{1}$ School of Medicine, Southeast University, National Clinical Research Center of Kidney Diseases, Jinling Hospital, Nanjing, China

${ }^{2}$ National Clinical Research Center of Kidney Diseases, Jinling Hospital, Nanjing University School of Medicine, Nanjing, China

${ }^{3}$ Department of Intelligent Clinical Decision Support, Ping An Healthcare Technology, Beijing, China

\section{ABSTRACT}

Introduction We assessed the association between guideline adherence and outcomes of clinical parameter control and end-stage kidney disease (ESKD), and further studied the effect of parameter control on ESKD for Chinese patients with diabetic nephropathy (DN). Research design and methods In this retrospective study, 1128 patients with DN (15,374 patient-visit samples) diagnosed by renal biopsy were enrolled. Samples were classified as adherence and nonadherence based on whether prescribed drugs conformed to medication regimen and drug contraindication recommended by guidelines, including American Diabetes Association (ADA) and Chinese guidelines. Guideline adherence rate was calculated on all samples for antihyperglycemic, antihypertensive and lipid-lowering treatments. Clinical parameter control was compared after 3-6 months' therapy between two groups by generalized estimating equation models. Time-dependent Cox models were applied to evaluate the influence of guideline adherence on ESKD. Latent class mixed model was used to identify distinct trajectories for parameters and their ESKD risks were compared using Cox proportional-hazards models.

Results Guideline adherence rate of antihyperglycemic therapy was the highest, with $72.87 \%$ and $68.15 \%$ of samples meeting ADA and Chinese guidelines, respectively. Adherence was more likely to have good glycated hemoglobin A1c (HbA1c) control (ADA: OR 1.46, $95 \% \mathrm{Cl} 1.12$ to 1.88 ; Chinese guideline: OR $1.42,95 \% \mathrm{Cl}$ 1.09 to 1.85) and good blood pressure control (ADA: OR $1.35,95 \% \mathrm{Cl} 1.03$ to 1.78 ; Chinese guideline: OR 1.39, $95 \% \mathrm{Cl} 1.08$ to 1.79) compared with nonadherence. The improvement of patient's adherence showed the potential to reduce ESKD risk. For proteinuria, low-density lipoprotein cholesterol (LDL-C), systolic blood pressure and uric acid, patients in higher-value trajectory group had higher ESKD risk. Proteinuria and LDL-C trajectories were most closely related to ESKD risk, while the risk was not significantly different in $\mathrm{HbA} 1 \mathrm{c}$ trajectories.

Conclusions Guideline adherence and good control of proteinuria and LDL-C in clinical practice are important and in need for improving clinical outcomes in patients with DN.

\section{INTRODUCTION}

Diabetic nephropathy (DN) is a chronic kidney disease (CKD) caused by diabetes mellitus, which is the main cause of end-stage

\section{Significance of this study}

What is already known about this subject?

> Adherence to guideline associates with improved clinical outcome in patients with type 2 diabetes with renal disease. However, there was no evidence for patients with diabetic nephropathy (DN) diagnosed by renal biopsy.

- The relationship between the longitudinal changes in clinical parameters and end-stage kidney disease (ESKD) risk was not clear.

What are the new findings?

- For patients with biopsy-proven DN, guideline adherence was associated with better glycemic and blood pressure control, and showed the potential to reduce ESKD risk by comprehensively assessing the antihyperglycemic, antihypertensive and lipidlowering treatments.

- The continuous control of proteinuria, low-density lipoprotein cholesterol (LDL-C), systolic blood pressure and uric acid were more likely to reduce ESKD risk, particularly in proteinuria and LDL-C.

- Glycated hemoglobin A1c level was not associated with ESKD risk.

How might these results change the focus of research or clinical practice?

- This study demonstrates that guideline adherence and good control of proteinuria and LDL-C in patients with DN are important in improving prognosis. It provides theoretical evidence to help in establishing the policies of standardized treatment for DN.

kidney disease (ESKD) at present. ${ }^{1}$ Approximately $20 \%-40 \%$ of patients with diabetes have progressed to DN. ${ }^{23}$ In China, the prevalence of $\mathrm{DN}$ is also increasing. ${ }^{4-6}$ In order to standardize the diagnosis and treatment of DN, multi-version clinical practice guidelines were formulated. ${ }^{7-11}$ These guidelines involved diagnosis, treatment goals, therapeutic strategies and so on. It was emphasized that patients with DN should adopt a 
comprehensive treatment of antihyperglycemic therapy, hypertension control and lipid management on the basis of changing their lifestyle in order to slow the progression of DN.

In theory, the development of various guidelines can help to guide the diagnosis and treatment of the clinician and improve the prognosis of patients. However, in actual practice, are the treatments of patients with $\mathrm{DN}$ in China accordant with guidelines? Is guideline adherence conducive to the improvement of clinical parameter control and long-term outcome? How about the association between longitudinal changes in clinical parameters and outcome in patients with DN? The interpretation of these questions will help improve the treatment strategies of patients with DN with more guideline-concordance and real-world evidence integrated, assisting clinicians with most effective prescriptions.

Some published studies assessed the adherence to guidelines for patients with type 2 diabetes with renal disease and the guideline was interpreted relatively simply. ${ }^{12-15}$ For example, Eder et $a l^{12}$ assessed the guideline adherence with respect to metabolic and blood pressure control, use of renin-angiotensin system-blocking agents, statins and acetylsalicylic acid for patients with type 2 diabetes with and without renal disease. Chen et $a l^{13}$ only assessed whether patients adjusted the dose of oral antidiabetic agents or avoided using them in patients with type 2 diabetes with moderate to severe CKD. Nevertheless, neither of the studies elaborated on the guideline-recommended medication regimen, namely which drug therapy should be chosen and how to combine drugs for precise patients grouped by their clinical conditions and medication history. Chen et $a l^{15}$ assessed the association between oral antidiabetic drug treatment concordance with guidelines and related economic and clinical outcomes in patients with type 2 diabetes and stage 3 to 5 CKD. However, there was no evidence for patients with DN diagnosed by renal biopsy. Also, they did not comprehensively assess the antihyperglycemic, antihypertensive and lipid-lowering treatments. As far as we know, there was no study to assess the effect of longitudinal trajectories in clinical parameter (ie, increasing, stable or decreasing) on ESKD for patients with DN.

Therefore, our study aimed to fill this gap. We first extracted the medication regimens and drug contraindications of patients with DN in American Diabetes Association (ADA) and Chinese guidelines, and further evaluated the guideline adherence to antihyperglycemic, antihypertensive and lipid-lowering treatments comprehensively. Then we analyzed the influence of guideline-concordant treatment on clinical outcomes in terms of short-term clinical parameter control and longterm ESKD occurrence. In addition, latent class mixed model was used to identify longitudinal trajectory groups for clinical parameters, and further it was studied how different trajectory groups were associated with ESKD risk in patients with $\mathrm{DN}$.

\section{RESEARCH DESIGN AND METHODS}

\section{Study population}

A total of 1906 patients with biopsy-proven DN in the Nanjing Diabetic Nephropathy Registry from January 2003 to December 2018 were reviewed. At our institution, the general indication for renal biopsy is persistent albuminuria or decreased serum creatinine, with a particular emphasis on sudden-onset overt proteinuria, obvious hematuria or rapidly progressive glomerular nephropathy. ${ }^{16}$ Patients meeting one of the following criteria were excluded: (1) patients with other renal diseases (such as membranous nephropathy and IgA nephropathy); (2) patients who had received kidney transplantation or dialysis before enrollment; (3) DN not caused by type 2 diabetes; (4) less than 14 days from enrollment to the occurrence of ESKD; (5) follow-up time was less than 1 year and no ESKD occurred; (6) patients without estimated glomerular filtration rate (eGFR) record. Finally, 1128 patients were enrolled with 15,374 follow-up visits from the enrollment time to outcome event (online supplementary figure $\mathrm{S} 1$ ).

\section{Clinical data collection}

The baseline and follow-up data of the patients were obtained from the Nanjing Diabetic Nephropathy Registry database. The time when a patient was diagnosed by renal biopsy was regarded as enrollment time, which was also baseline time. Eligible patients were followed up from the time of biopsy until the earliest outcome event (patient transferred out, death, study end date, ESKD occurrence). The follow-up visits of these patients were performed two to four times per year based on the patient's individual condition. At baseline, demographic information (eg, sex and age) and disease history (eg, diabetes and atherosclerotic cardiovascular disease (ASCVD) ) were collected. At baseline and each follow-up visit, laboratory test results, current and previous medications in prescriptions were collected. The laboratory test results included glycated hemoglobin A1c (HbAlc), blood pressure (BP), uric acid (UA), proteinuria (PRO), serum creatine ( $\mathrm{SCr}$ ), low-density lipoprotein cholesterol (LDL-C) and so on, and the medications in prescriptions included antihyperglycemic drugs, antihypertensive drugs and lipid-lowering drugs.

The eGFR was estimated using the chronic kidney disease epidemiology collaboration (CKD-EPI) formula. ${ }^{17}$ $\mathrm{ESKD}$ was defined as eGFR $<15 \mathrm{~mL} / \mathrm{min} / 1.73 \mathrm{~m}^{2}$ for over 3 months, with initiation of dialysis or transplantation.

\section{Selection of guidelines}

In this study, multi-version guidelines including ADA guideline for $2018^{8}$ and Chinese guideline ${ }^{9}$ were selected for comparative analysis. In addition, some drugs metabolized or excreted in the kidney, so in patients with renal insufficiency, the decrease of renal excretion or the clearance of its active metabolites can lead to adverse reactions such as hypoglycemia. ${ }^{18}$ Therefore, another two national guidelines supplemented the drug contraindications 
when eGFR was below $60 \mathrm{~mL} / \mathrm{min} / 1.73 \mathrm{~m}^{2}$ for Chinese guideline in this study. ${ }^{10}{ }^{11}$ Treatment strategies in domestic and international guidelines were summarized in online supplementary table S1.

\section{Statistical analysis}

In this study, the guideline-concordant evaluation and associated clinical outcomes were given at both sample level and patient level. We defined a sample as a follow-up visit merged with diagnosis, laboratory tests and treatments (online supplementary figure S1B), so there were multiple samples for one patient. Taking the antihyperglycemic therapy of ADA guideline for example, the flowchart of sample inclusion when evaluating the influence of guideline adherence on short-term/longterm outcome is shown in online supplementary figure S2. The following analysis was performed separately for domestic and international guidelines.

The guideline adherence rate was calculated at sample level. For each kind of guideline, we partitioned all samples into adherence and nonadherence based on whether the type and number of prescribed medications conformed to guideline recommendations and the drugs that should be completely avoided at low eGFR were discontinued. The short-term clinical outcomes were defined as target achievement rate of the key parameters, namely the percentages of samples with (1) HbA1c < 7\%, (2) both systolic BP <130 and diastolic BP $<80 \mathrm{~mm} \mathrm{Hg}$, and (3) LDL-C $<1.8 \mathrm{mmol} / \mathrm{L}$ for very-highrisk, LDL-C $<2.6 \mathrm{mmol} / \mathrm{L}$ for high-risk (very-high-risk: have a definite history of ASCVD; high-risk: diabetic patients without history of ASCVD). Then we compared these clinical outcomes in short term (after 3-6 months' therapy) between the two groups. The generalized estimating equation (GEE) models with an exchangeable correlation structure were used to account for correlation between samples within the same patient when evaluating the association between guideline-concordant treatment and short-term clinical outcomes. ${ }^{19}$ The models were also performed to control potential confounding factors including age, sex, eGFR and corresponding parameters (HbA1c or systolic BP, diastolic BP or LDL-C, triglyceride (TG) ) measured at baseline or current visit. In order to evaluate the influence of guideline versions published in different years, we divided ADA guidelines from 2003 to 2018 into five groups according to the similarity in the antihypertensive treatment regimen and BP control target (online supplementary table S2). The 2007, 2012, 2014, 2016 and 2018 versions of ADA were selected as representative of each group to study the association between guideline adherence and BP-goal achieving rate.

Then, we performed the long-term effect evaluation results at patient level. For each patient, we first computed its patient's adherence rate with regard to antihyperglycemic therapy, hypertension control and lipid management, respectively, through dividing the number of guideline-adherent visits by the total number of visits, that is, to quantify the extent to which each patient complied with the guidelines. In the whole population, we further illustrated the relationship between patient's adherence rate and ESKD occurrence rate. To avoid the sharp variability in less visit number, patients with a visit number of 5 or greater were selected to evaluate the long-term clinical outcome. In addition, to evaluate the influence of exposing to different guidelines, taking hypertension control of ADA guideline for an example (online supplementary table S2), we illustrated the relationship between patient's adherence rate and ESKD occurrence rate based on multi-version ADA guidelines that the different visits of the patient were exposed to.

Considering that the adherence for a patient to 2018 version of guidelines was time varying and the follow-up time for different patients were varied, the timedependent Cox models were used to evaluate the influence of guideline adherence on long-term outcome of ESKD. ${ }^{20}$ The models were adjusted for sex, age, eGFR, duration of diabetes and time-dependent covariates (HbA1c, systolic BP, diastolic BP, LDL-C, TG, PRO, UA).

\section{Trajectory analyses}

In order to illustrate how changes in clinical parameters were associated with the prognosis of patients with $\mathrm{DN}$, latent class mixed model (LCMM) was used to identify distinct longitudinal trajectory groups for each key clinical parameter using R package "lcmm". ${ }^{21}$ LCMM enables agnostic detection of multiple subpopulations with distinct longitudinal patterns of clinical parameter. ${ }^{22} 23$ Compared with the traditional method, trajectory analyses may be more beneficial to identify dynamic patterns over time for laboratory test records in their individual patients rather than average changes in all of their patients. Thus, we used LCMM to fit the longitudinal records with different numbers of trajectory groups in various polynomial (linear, quadratic and cubic) terms. Best models were chosen with the following key criteria: sufficient patient number in each class and relatively low Bayesian Information Criterion. Furthermore, Cox proportional-hazards models were applied to analyze how different trajectory groups were associated with the occurrence of ESKD.

Potential confounding factors were adjusted stepwise in each Cox model. Model 1 adjusted for demographic factors (age and sex). Model 2 further adjusted for the visit number during the follow-up period and the laboratory test results (including $\mathrm{HbAlc}$, systolic $\mathrm{BP}$, diastolic BP, TC, TG, LDL-C, HDL-C, UA) measured at baseline. Model 3 further adjusted for the baseline eGFR. In order to determine whether the results of the study were affected by changing the follow-up time, we performed the sensitivity analysis by changing the inclusion time span of the trajectory and repeating the aforementioned analysis. All $\mathrm{p}$ values were two tailed, and $\mathrm{p}$ value $<0.05$ was considered statistically significant. 
Table 1 Baseline characteristics of study participants

\begin{tabular}{ll}
\hline Baseline characteristics & Values \\
\hline Age, years & $50.17 \pm 9.37$ \\
\hline Male sex & $778(69 \%)$ \\
\hline Duration of diabetes, years & $9.13 \pm 6.56$ \\
\hline HbA1c, \% & $6.70 \pm 1.23$ \\
\hline Uric acid, $\mu \mathrm{mol} / \mathrm{L}$ & $388.43 \pm 96.21$ \\
\hline Total cholesterol, mmol/L & $5.22 \pm 1.71$ \\
\hline HDL-C, mmol/L & $1.13 \pm 0.39$ \\
\hline LDL-C, mmol/L & $3.11 \pm 1.35$ \\
\hline Triglyceride, mmol/L & $1.55(1.11-2.14)$ \\
\hline Systolic BP, mm Hg & $136.89 \pm 20.83$ \\
\hline Diastolic BP, mm Hg & $78.25 \pm 10.88$ \\
\hline Proteinuria, g/24h & $1.59(0.71-3.35)$ \\
\hline Serum creatine, $\mathrm{mg} / \mathrm{dL}$ & $1.29(0.89-1.86)$ \\
\hline eGFR (mL/min/1.73 m²) & $58.98(38.36-92.03)$ \\
\hline Follow-up, years & $3.26(1.56-5.53)$ \\
\hline Anemia, \% & 49.91 \\
\hline ASCVD, \% & 16.9 \\
\hline
\end{tabular}

Values for continuous variables given as mean \pm SD or median (IQR); for categorical variables, as count (percentage). ASCVD, atherosclerotic cardiovascular disease; BP, blood pressure; eGFR, estimated glomerular filtration rate; HbA1c, hemoglobin A1c; HDL-C, high-density lipoprotein cholesterol; LDL-C, low-density lipoprotein cholesterol.

\section{RESULTS}

Baseline characteristics

Of the 1128 patients enrolled in the study, 778 were male $(69 \%)$. At the time of biopsy, the mean age was $50.17 \pm 9.37$ years and the mean duration of diabetes was $9.13 \pm 6.56$ years. The median follow-up period was 3.26 (IQR, 1.56-5.53) years. The median baseline serum creatinine level was 1.29 (IQR, 0.89-1.86) $\mathrm{mg} / \mathrm{dL}$ and the median eGFR was 58.98 (IQR, 38.36-92.03) mL/ $\min / 1.73 \mathrm{~m}^{2}$ (table 1 ). The ADA guideline adherence rates at baseline for antihyperglycemic, antihypertensive and lipid-lowing treatment were $55.10 \%, 42.09 \%$ and $30.54 \%$, respectively. The Chinese-guideline adherence rates at baseline were $43.88 \%, 45.15 \%$ and $19.63 \%$, respectively.

\section{Adherence to guidelines and achievement of control targets}

As shown in table 2, the overall guideline adherence rate for antihyperglycemic therapy was the highest. ADA-guideline adherence rate and Chinese-guideline adherence rate for antihyperglycemic therapy were $72.87 \%$ and $68.15 \%$, respectively. Since ADA guideline just mentioned drug contraindications for antihyperglycemic therapy but not for hypertension control and lipid management, we just evaluated the compliance with the medication regimens in $\mathrm{ADA}$ guideline for antihypertensive and lipid-lowering treatment. For antihypertensive treatment, ADA-guideline adherence rate and Chinese-guideline adherence rate were $36.00 \%$ and $37.78 \%$, respectively. ADA-guideline adherence rate and Chinese-guideline adherence rate for lipid management were $36.98 \%$ and $28.97 \%$, respectively.

The short-term clinical outcomes between adherence and nonadherence were compared in table 3. After confounder adjustment by GEE models, for both ADA and Chinese guidelines, the adherence was more likely to have good HbA1c control (ADA: OR 1.46, 95\% CI 1.12 to 1.88 ; Chinese guideline: OR $1.42,95 \%$ CI 1.09 to 1.85$)$, good blood pressure control (ADA: OR 1.35, 95\% CI 1.03 to 1.78; Chinese guideline: OR $1.39,95 \%$ CI 1.08 to 1.79 ) than nonadherence. Since there is no specified control goal of blood lipid in ADA guideline, the lipid-goal achieving rate was not assessed for ADA guideline. For Chinese guideline, we found no statistically significant difference between adherence and nonadherence (OR $1.18,95 \%$ CI 0.85 to 1.62$)$.

The guideline adherence and associated BP-goal achieving rate are shown based on guidelines published in different years in online supplementary table S3. There is an irregularity between the guideline adherence rate and guideline version, as the guideline adherence rate with ADA guideline in 2016 was the highest $(55.12 \%)$. Interestingly, the BP-goal achieving rate is closely related to the BP control target. The BP-goal achieving rate if concordant with ADA guideline in 2016 was the highest (55.71\%) due to a relatively high goal of $140 / 90 \mathrm{~mm} \mathrm{Hg}$. We also found among the five versions of ADA guidelines that guideline-adherent treatments tend to have better BP-goal achieving rate than nonadherence.

\section{Adherence to guidelines and the risk of ESKD}

The curves in A, B, E and F were with an obvious downward trend as a whole (online supplementary figure S3), which means that the higher the patient's adherence rate, the lower the ESKD occurrence rate. It was indicated that the improvement of patient's adherence showed the potential to reduce ESKD risk. Although the curve was fluctuated in online supplementary figure S3D, the whole curve still showed a slight downward trend. While in online supplementary figure S3C the curve was fluctuated horizontally, it was indicated that there was no obvious trend in illustrating the relationship between the patient's adherence rate and ESKD occurrence rate for lipid management of ADA guideline. In addition, similar trends have been found in patients with a visit number of 5 or greater (online supplementary figure S4) and multiversion $\mathrm{ADA}$ guidelines-based adherence rate (online supplementary figure S5).

The influence of guideline adherence on ESKD was evaluated using time-dependent Cox models (table 4). After adjustment for confounders, adherence to Chinese guideline was associated with a decrease in ESKD risk for antihyperglycemic therapy (adjusted HR 0.74; $95 \%$ CI 0.58 to $0.95 ; \mathrm{p}=0.016$ ), hypertension control (adjusted HR $0.66 ; 95 \%$ CI 0.50 to $0.87 ; \mathrm{p}=0.004$ ) and 
Table 2 ADA and Chinese guideline adherence rate for antihyperglycemic therapy, hypertension control and lipid management in patients with diabetic nephropathy

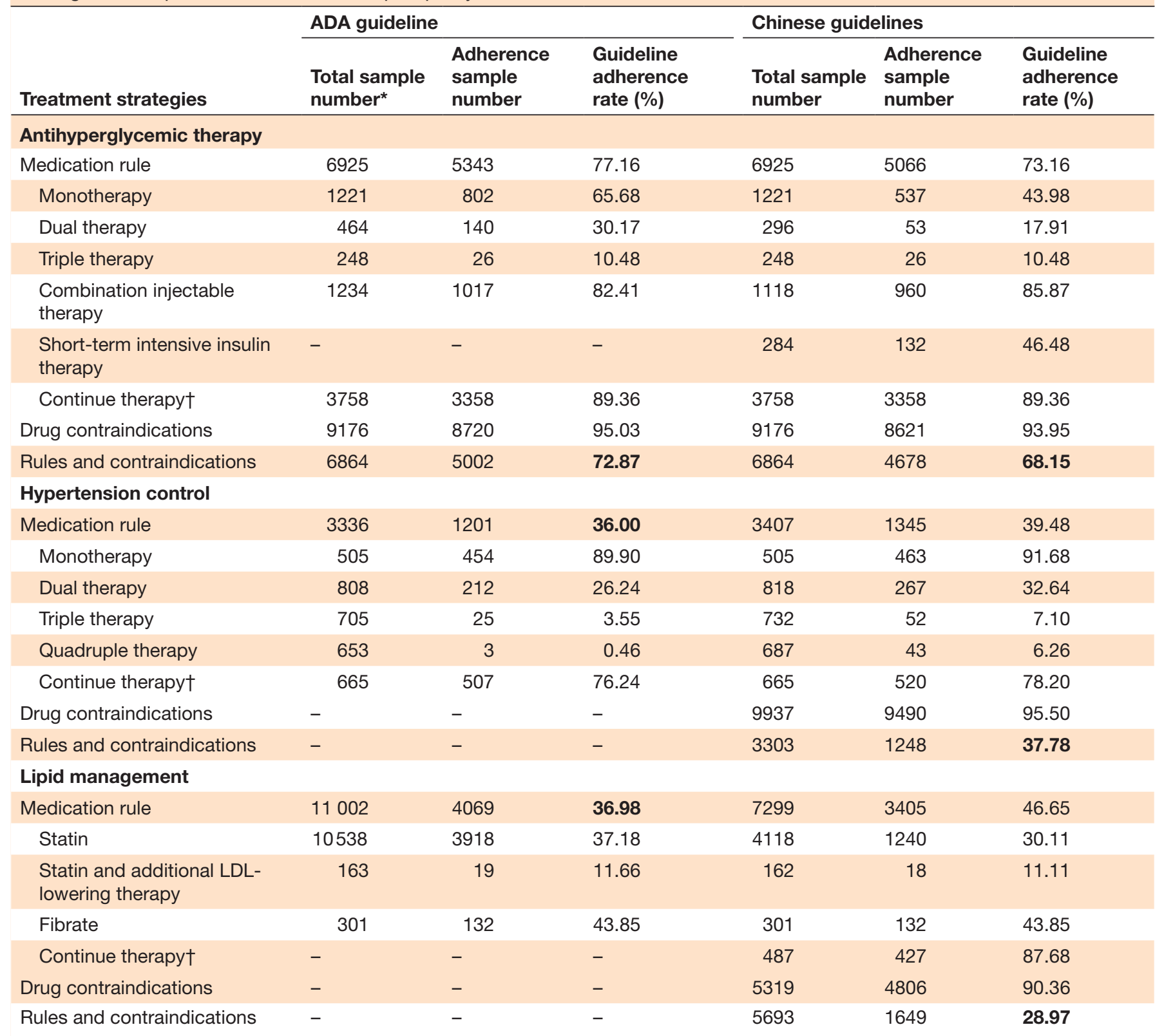

Bold values denote overall adherence rate of guidelines.

${ }^{*}$ The total sample number of each group represented the number of patient visits meeting the situation mentioned in corresponding rule in online supplementary table S1. The adherence sample number indicated the number of patient visits in which prescribed medications conformed to guideline-rule recommendations. Thus, guideline adherence rate was calculated through dividing the adherence sample number by the total sample number.

†Continue therapy: continued previous treatment when tolerated and target achieved.

ADA, American Diabetes Association.

lipid management (adjusted HR 0.52; $95 \%$ CI 0.39 to $0.71 ; \mathrm{p}<0.001)$, while adherence to ADA guideline was associated with a decrease in ESKD risk for antihyperglycemic therapy (adjusted HR $0.71 ; 95 \%$ CI 0.55 to 0.9 ; $\mathrm{p}=0.006$ ). However, no significant association existed between adherence to ADA guideline and ESKD risk for hypertension control (adjusted HR 0.89; 95\% CI 0.66 to $1.21 ; \mathrm{p}=0.471$ ) and lipid management (adjusted HR $0.95 ; 95 \%$ CI 0.76 to $1.19 ; \mathrm{p}=0.658$ ).
Longitudinal trajectory of key clinical parameters and the risk of ESKD

The median number of follow-up visits was 9.0 (IQR 5.0-16.0) and median follow-up time is 3.26 (IQR 1.56$5.53)$ years. During the first 2 years of follow-up, about $70 \%$ of participants did not develop ESKD in the study. Therefore, we performed the longitudinal trajectory analysis on the key parameters in the first 2 years from the enrollment, and analyzed the association between 


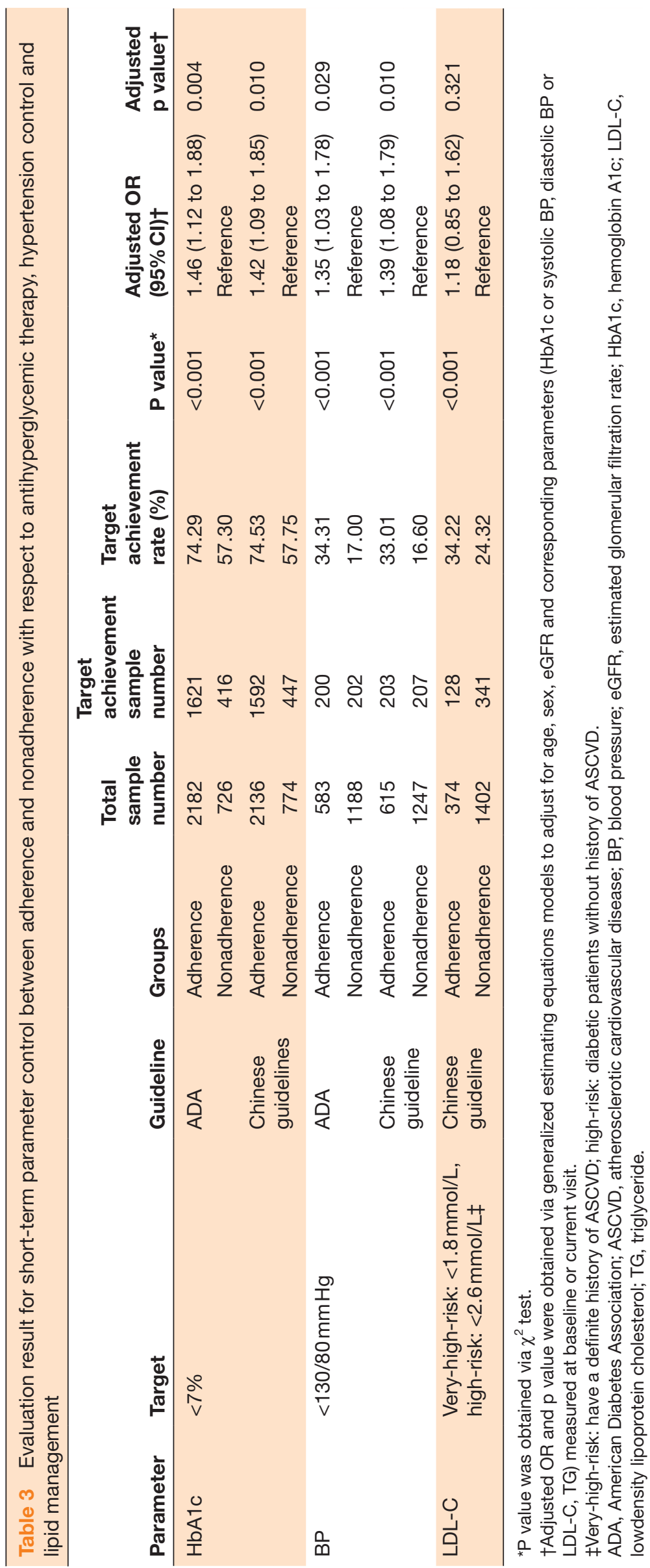

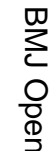

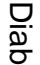

गु

๑ి

$\overrightarrow{\overrightarrow{\mathrm{C}}}$

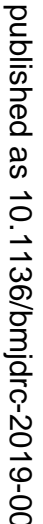

官

욱

$\vec{G}$

N

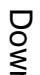


Table 4 Associations between guideline adherence and ESKD risk using time-dependent Cox models

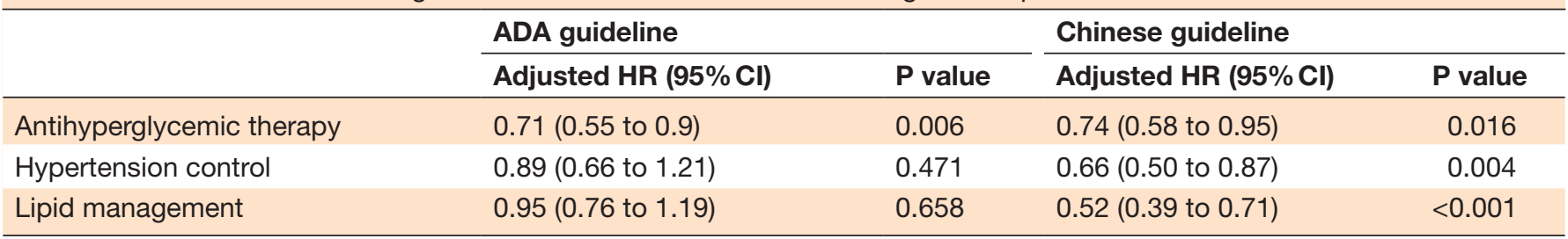

Adjusted HR and $p$ value were obtained via time-dependent Cox models to adjust for sex, age, eGFR, duration of diabetes and timedependent covariates (HbA1c, systolic BP, diastolic BP, LDL-C, TG, PRO, UA).

ADA, American Diabetes Association; BP, blood pressure; eGFR, estimated glomerular filtration rate; ESKD, end-stage kidney disease; $\mathrm{HbA1c}$, hemoglobin A1c; LDL-C, low-density lipoprotein cholesterol; PRO, proteinuria; TG, triglyceride; UA, uric acid.

different trajectory groups and ESKD occurrence from the third year.

The distinct trajectory groups of each clinical parameter are shown in figure 1 . For proteinuria, we identified four distinct trajectories marked with Class 1-4 orderly: low stable (in black), moderate stable (in red), rising (in green) and declining (in blue) (figure 1A). After trajectory grouping, we compared their ESKD risk using Cox proportional-hazards models. Compared with lowstable proteinuria trajectory group (PRO-Class 1), the full multivariable-adjusted HR of ESKD was 8.29 (95\% CI 4.81 to $14.28, \mathrm{p}<0.001)$ for the rising proteinuria trajectory group (PRO-Class 3 ) and 7.46 (95\% CI 3.45 to 16.14 , $\mathrm{p}<0.001)$ for the declining proteinuria trajectory group (PRO-Class 4), as described in figure $1 \mathrm{~F}$ and online supplementary table S4 (model 3). It was indicated that compared with low-stable trajectory group (PRO-Class $1)$, participants with rising proteinuria trajectory group (PRO-Class 3) were at 8.29-fold higher risk of ESKD and participants with declining proteinuria trajectory group (PRO-Class 4) were at 7.46-fold higher risk of ESKD in fully adjusted analyses. For $\mathrm{HbAlc}$, four distinct trajectory groups were identified and illustrated in figure 1E. Patients with DN with higher HbAlc trajectory did not have a higher risk of ESKD ( $>00.05)$ compared with those with a low-stable $\mathrm{HbA1c}$ trajectory. For other parameters including LDL-C, systolic blood pressure (SBP) and UA, the results for the full multivariable-adjusted HR of ESKD showed that participants with higher trajectory were associated with higher risk of ESKD (figure 1B-D). Rising PRO trajectory (PRO-Class 3) and EX-high unstable LDL-C trajectory (LDL-Class 4) were most closely
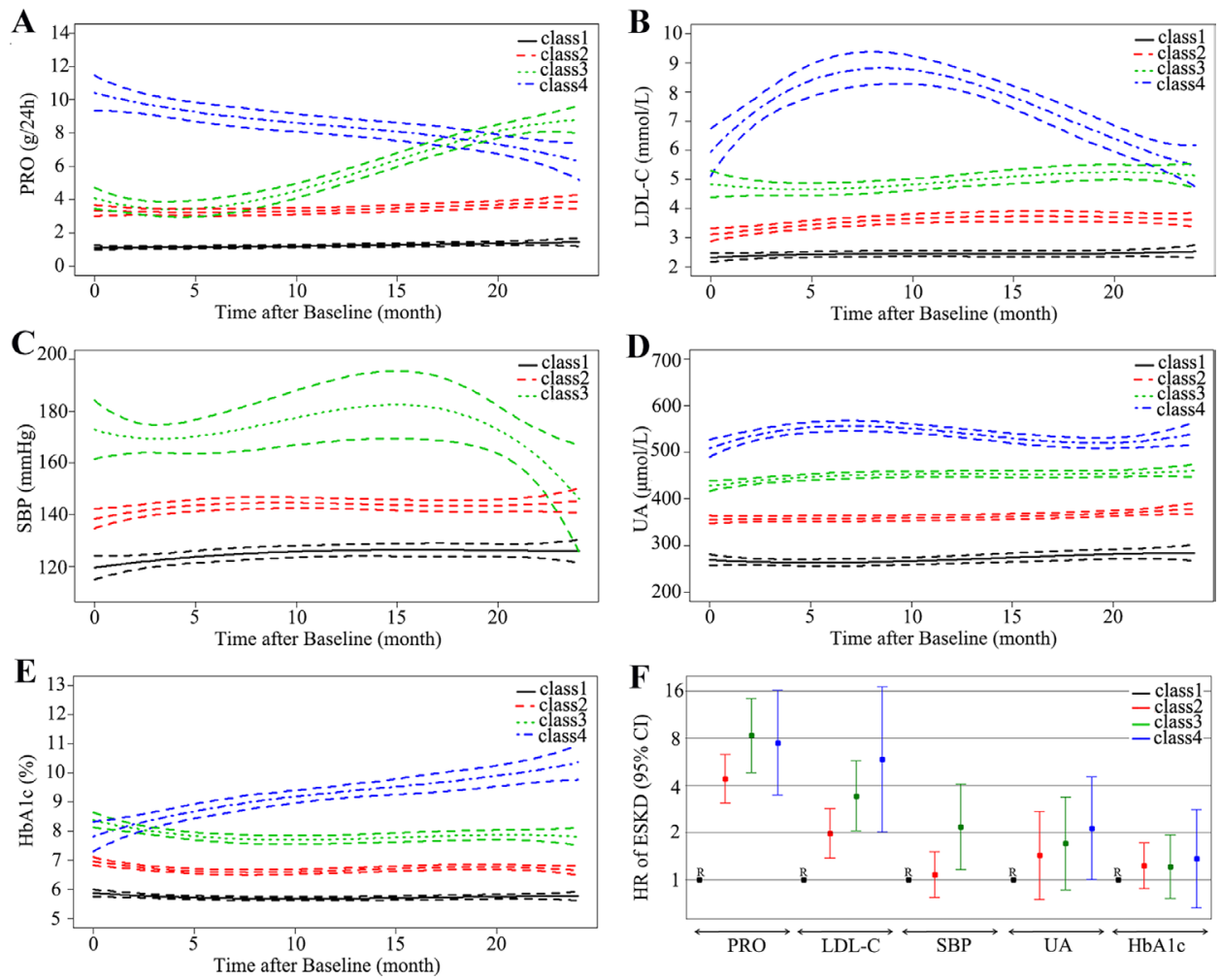

Figure 1 Trajectory modeling identified multiple distinct trajectory groups with $95 \% \mathrm{Cls}$ (between dashed lines) for PRO $(A), L D L-C(B), S B P(C), U A(D), H b A 1 c(E)$. The ESKD risks were compared according to trajectory groups of those key clinical parameters with fully adjusted HRs (low-stable trajectory as reference) (F). ESKD, end-stage kidney disease; HbA1c, hemoglobin A1c; LDL-C, low-density lipoprotein cholesterol; PRO, proteinuria; SBP, systolic blood pressure; UA, uric acid. 
associated with increased risk of ESKD (figure $1 \mathrm{~F}$ ). In summary, except $\mathrm{HbAlc}$, the continuous control of PRO, LDL-C, SBP and UA was more likely to reduce ESKD risk, particularly in PRO and LDL-C. The results of sensitivity analysis by changing the inclusion time span of the trajectory from 2 years to 3 years were similar (online supplementary table S5).

\section{DISCUSSION}

To our knowledge, this is the first study to assess guidelineconcordant treatment and associated clinical outcomes in patients with DN diagnosed by renal biopsy. The guideline adherence rates of $\mathrm{ADA}$ and Chinese guideline were similar. However, guideline adherence of treatments for patients with DN in China still needs to be improved, especially for antihypertensive and lipid-lowing treatment. We found that guideline-concordant treatments were associated with improved clinical outcomes, achieving good control of blood glucose and BP and showing the potential to reduce ESKD risk in the long term. Except HbA1c, the longitudinal trajectory analysis of PRO, LDL-C, SBP and UA indicated that good continuous control of these key parameters was associated with reduced ESKD risk, particularly for PRO and LDL-C.

Some previous study found guideline-concordant treatment was associated with better clinical outcomes. ${ }^{15} 24$ Similarly, we found that adherence to Chinese guideline was associated with a decrease in ESKD risk for antihyperglycemic therapy, hypertension control and lipid management among Chinese patients with DN. However, it seems that no significant association existed between adherence to ADA guideline and ESKD risk for hypertension control and lipid management in our study. It may be because that ADA guideline just mentioned drug contraindications for antihyperglycemic therapy but not for hypertension control and lipid management. ${ }^{8}$ So we just evaluated the compliance with the medication regimens in ADA guideline for antihypertensive and lipid-lowering treatment. Some patients in the adherence group tend to have higher ESKD risk if taking those drugs that should be discontinued at low eGFR, which probably leads to no significant difference in ESKD risk between adherence and nonadherence for hypertension control and lipid management of ADA guideline.

Proteinuria is an established marker of kidney damage, a risk factor for progression of CKD and a potent risk factor for mortality among the diabetic population. ${ }^{25}{ }^{26}$ Our results are in agreement, and it was found that proteinuria was most closely related to ESKD risk among those clinical parameters, and the prognosis could be improved by continuous good control of proteinuria. In addition, previous studies showed that the level of BP, lipid and UA were closely related to the progression of kidney disease in patients with DN. ${ }^{27-30}$ This was consistent with the longitudinal trajectory results of LDL-C, SBP and UA in this study, and all of them showed that patients in the trajectory group with higher value were associated with higher ESKD risk.

Our study showed that no difference in ESKD risk was observed among HbAlc trajectory groups. Studies exploring the impact of glycemic control on ESKD are limited and yield somewhat inconsistent results. Several studies have similar results to our study, indicating that lowering blood glucose did not appreciably reduce the incidence of ESKD. ${ }^{31-33}$ We discuss the possible reason in our study as follows. In the ADVANCE-ON study, to analyze the effect of intensive glucose control on ESKD by baseline CKD status, participants were divided into those with CKD stage 1 , stage 2 and stage $\geq 3 .{ }^{34}$ A graded reduction in the strength of the effect was seen as CKD stage increased and intensive glucose control did not reduce the incidence of ESKD in participants with CKD stage $\geq 3$. In our study, the proportion of participants with CKD stage $\geq 3$ was $39 \%$, which was quite higher than some studies that have similar results with our study, like VADT $(14 \%)$ and UKPDS (10\%) studies. ${ }^{35}$ This may be a possible reason why HbAlc level was not associated with ESKD risk. In addition, in patients with CKD, lower or higher HbAlc levels may be observed than would be expected from measured glucose control $^{36}$ because HbAlc is influenced by many factors including the lifespan of red blood cells, recombinant human erythropoietin, blood transfusions, iron supplements and anemia. ${ }^{37-40}$ Those interfering and confounding factors may lead to erroneous HbAlc values. Glycated albumin was not influenced by those factors, which may replace HbAlc to further explore the relationship between glycemic control and ESKD in patients with DN. ${ }^{37} 41$

The strengths of this study are twofold. First, the data of patients with DN used for adherence and effectiveness evaluation are of good quality. They consist of the medical records for a large number of Chinese patients with DN and cover a long time span with median follow-up time of 3.26 (IQR 1.56-5.53) years. Second, the methods to investigate the influence of guideline adherence and parameter control on the clinical outcomes are comprehensive, including GEE models, Cox proportional-hazards models and latent class mixed model. We consider the guideline adherence of three types of treatments (antihyperglycemic, antihypertensive and lipid-lowing therapy) in both ADA and Chinese guidelines, and evaluate two types of outcomes (the control of clinical parameters in the short term and the occurrences of ESKD in the long term). In this study, considering the correlation between visit samples within the same patient, guideline adherence and its effect on control target achievement of parameters were evaluated through GEE models. Furthermore, considering that adherence to a specific guideline for a patient was time varying and the follow-up time for different patients was varied, the time-dependent Cox models have been applied to solve this problem and evaluate the influence of guideline adherence on long-term outcome of ESKD. Finally, to investigate the effect of parameter 
control on ESKD, an advanced method of LCMM was applied to identify the trajectory groups in clinical parameters at patient level, and associated ESKD risks in different trajectory groups were compared using Cox proportional-hazards models with sensitivity analysis.

However, our study has its limitations. First, it was a single-center retrospective study and the selection bias may exist. Some patients with incomplete follow-up data were not included in the study. Second, since the information of drug dosage was incomplete, we did not consider the drug dosage adjustment in patients with DN. Also, the incomplete death records did not allow us to build up competitive risk models. Third, we only measure guideline adherence without any measures of patients' adherence to prescription. Finally, although the guidelines increasingly emphasize personalized treatment, in this study unified therapeutic targets were selected (eg, HbAlc $<7 \%$ ) without considering personalized control targets for individual patients.

In conclusion, the results in this retrospective study suggested that adherence to guidelines can help patients with DN patients to reach therapeutic targets and improve long-term prognosis. At the same time, the importance of strict control of proteinuria and LDL-C in patients with $\mathrm{DN}$ was emphasized. It provides the real-world evidence to help in establishing the policies of standardized treatment for DN in China.

Acknowledgements The authors thank all volunteers for their generous participation in this study and thank Haitao Zhang, Yongchun Ge and Song Jiang (National Clinical Research Center of Kidney Diseases, Jinling Hospital, Nanjing University School of Medicine, Nanjing, China) for valuable suggestions on data analysis.

Contributors $\mathrm{JL}$ and WZ wrote the manuscript and contributed to data analysis and discussion. TC and XS contributed to the discussion and reviewed the manuscript. ZX performed the analyses and reviewed the manuscript. $H X, Y A, C Z$, $\mathrm{GH}$ and $\mathrm{GX}$ contributed to data collection and edited the manuscript. ZL designed the study and oversaw all clinical aspects of study conduct and manuscript preparation. ZL is the guarantor of this work and, as such, had full access to all the data in the study and takes responsibility for the integrity of the data and the accuracy of the data analysis.

Funding This study was supported by Key project of Social Development Program of Jiangsu Province (BE2016747) and National Key Research and Development Program of China (2016YFC0901202).

Competing interests None declared.

Patient consent for publication Not required.

Ethics approval This study followed the tenets of the Declaration of Helsinki and was approved by the ethics committee of the Nanjing General Hospital (2019NZKYKS-008-01), Nanjing, China.

Provenance and peer review Not commissioned; externally peer reviewed.

Data availability statement No data are available.

Open access This is an open access article distributed in accordance with the Creative Commons Attribution Non Commercial (CC BY-NC 4.0) license, which permits others to distribute, remix, adapt, build upon this work noncommercially, and license their derivative works on different terms, provided the original work is properly cited, appropriate credit is given, any changes made indicated, and the use is non-commercial. See: http://creativecommons.org/ licenses/by-nc/4.0/.

\section{REFERENCES}

$1 \mathrm{KDOQI}$. KDOQI clinical practice guidelines and clinical practice recommendations for diabetes and chronic kidney disease. Am J Kidney Dis 2007;49:S12-154.

2 Tuttle KR, Bakris GL, Bilous RW, et al. Diabetic kidney disease: a report from an ADA consensus conference. Diabetes Care 2014;37:2864-83.

3 de Boer IH, Rue TC, Hall YN, et al. Temporal trends in the prevalence of diabetic kidney disease in the United States. JAMA 2011;305:2532-9.

4 Zhang L, Long J, Jiang W, et al. Trends in chronic kidney disease in China. N Engl J Med 2016;375:905-6.

5 Liu Z-H. Nephrology in China. Nat Rev Nephrol 2013;9:523-8.

6 Hou J-H, Zhu H-X, Zhou M-L, et al. Changes in the spectrum of kidney diseases: an analysis of 40,759 biopsy-proven cases from 2003 to 2014 in China. Kidney Dis 2018;4:10-19.

$7 \mathrm{KDIGO}$. Clinical practice guideline for the evaluation and management of chronic kidney disease. Kidney Int Suppl 2012;2013:1-150.

8 American Diabetes Association. Standards of medical care in diabetes-2018. Diabetes Care 2018;41:S11-153.

9 Chinese Diabetes Society. Chinese guideline for the prevention and treatment of type 2 diabetes mellitus (2017 edition). Chin J Diabetes Mellitus 2018;10:4-67.

10 The Microvascular Complications Group of Chinese Diabetes Association. Chinese clinical practice guideline of diabetic kidney disease. Chin J Diabetes Mellitus 2019;11:15-28.

11 Chinese Endocrinologist Association, Chinese Medical Doctor Association. Application principle for oral glucose-lowering drugs in type 2 diabetes mellitus patients with chronic kidney disease: Chinese experts consensus: 2015 update. Chin J Endocrinol Metab 2016;32:455-60.

12 Eder S, Leierer J, Kerschbaum J, et al. Guidelines and clinical practice at the primary level of healthcare in patients with type 2 diabetes mellitus with and without kidney disease in five European countries. Diab Vasc Dis Res 2019;16:47-56.

13 Chen S-Y, Siu K, Kovacs B, et al. Clinical and economic outcomes associated with National Kidney Foundation guideline-concordant oral antidiabetic drug treatment among type 2 diabetes patients with chronic kidney disease. Curr Med Res Opin 2012;28:493-501.

14 Wu N, Yu X, Greene M, et al. Evaluation of the prevalence of chronic kidney disease and rates of oral antidiabetic prescribing in accordance with guidelines and manufacturer recommendations in type 2 diabetic patients within a long-term care setting. Int J Nephrol 2014;2014:1-10.

15 Chen S-Y, Lee Y-C, Alas V, et al. Outcomes associated with nonconcordance to National Kidney Foundation guideline recommendations for oral antidiabetic drug treatments in patients with concomitant type 2 diabetes and chronic kidney disease. Endocr Pract 2014;20:221-31.

16 An Y, Xu F, Le W, et al. Renal histologic changes and the outcome in patients with diabetic nephropathy. Nephrol Dial Transplant 2015;30:257-66.

17 Levey AS, Stevens LA, Schmid CH, et al. A new equation to estimate glomerular filtration rate. Ann Intern Med 2009;150:604-12.

18 Yale J-F. Oral antihyperglycemic agents and renal disease: new agents, new concepts. J Am Soc Nephrol 2005;16 Suppl 1:S7-10.

19 Hardin JW, Hilbe JM. Generalized estimating equations. New York: Chapman and Hall, 2003.

20 Fisher LD, Lin DY. Time-dependent covariates in the Cox proportional-hazards regression model. Annu Rev Public Health 1999;20:145-57.

21 Proust-Lima C, Philipps V, Liquet B. Estimation of extended mixed models using latent classes and latent processes: the $R$ Package Icmm. J Stat Softw 2017;78:1-56.

22 Allen NB, Siddique J, Wilkins JT, et al. Blood pressure trajectories in early adulthood and subclinical atherosclerosis in middle age. JAMA 2014;311:490-7.

23 Song M, Hu FB, Wu K, et al. Trajectory of body shape in early and middle life and all cause and cause specific mortality: results from two prospective US cohort studies. BMJ 2016;353:i2195.

24 Karlsson SA, Eliasson B, Franzén S, et al. Risk of cardiovascular event and mortality in relation to refill and guideline adherence to lipid-lowering medications among patients with type 2 diabetes mellitus in Sweden. BMJ Open Diabetes Res Care 2019;7:e000639.

25 Ying T, Clayton P, Naresh C, et al. Predictive value of spot versus 24-hour measures of proteinuria for death, end-stage kidney disease or chronic kidney disease progression. BMC Nephrol 2018;19:55.

26 Astor BC, Matsushita K, Gansevoort RT, et al. Lower estimated glomerular filtration rate and higher albuminuria are associated with 
mortality and end-stage renal disease. A collaborative meta-analysis of kidney disease population cohorts. Kidney Int 2011;79:1331-40.

27 Almquist T, Jacobson SH, Mobarrez F, et al. Lipid-lowering treatment and inflammatory mediators in diabetes and chronic kidney disease. Eur J Clin Invest 2014;44:276-84.

28 Bakris GL, Williams M, Dworkin L, et al. Preserving renal function in adults with hypertension and diabetes: a consensus approach. National Kidney Foundation Hypertension and Diabetes Executive Committees Working Group. Am J Kidney Dis 2000;36:646-61.

29 Patney V, Chaudhary K, Whaley-Connell A. Treatment of diabetic kidney disease with hypertension control and renin angiotensin system inhibition. Adv Chronic Kidney Dis 2018;25:158-65.

30 De Cosmo S, Viazzi F, Pacilli A, et al. Serum uric acid and risk of CKD in type 2 diabetes. Clin J Am Soc Nephrol 2015;10:1921-9.

31 Duckworth W, Abraira C, Moritz T, et al. Glucose control and vascular complications in veterans with type 2 diabetes. $N$ Engl $J$ Med 2009;360:129-39.

32 Ismail-Beigi F, Craven T, Banerji MA, et al. Effect of intensive treatment of hyperglycaemia on microvascular outcomes in type 2 diabetes: an analysis of the ACCORD randomised trial. Lancet 2010;376:419-30.

33 Limkunakul C, de Boer IH, Kestenbaum BR, et al. The association of glycated hemoglobin with mortality and ESKD among persons with diabetes and chronic kidney disease. J Diabetes Complications 2019;33:296-301.
34 Wong MG, Perkovic V, Chalmers $\mathrm{J}$, et al. Long-term benefits of intensive glucose control for preventing end-stage kidney disease: ADVANCE-ON. Diabetes Care 2016;39:694-700.

35 Zoungas S, Arima H, Gerstein HC, et al. Effects of intensive glucose control on microvascular outcomes in patients with type 2 diabetes: a meta-analysis of individual participant data from randomised controlled trials. Lancet Diabetes Endocrinol 2017:5:431-7.

36 Rodríguez-Segade S, Rodríguez J, García López JM, et al. Intrapersonal $\mathrm{HbA}(1 \mathrm{c})$ variability and the risk of progression of nephropathy in patients with Type 2 diabetes. Diabet Med 2012;29:1562-6.

37 Vos FE, Schollum JB, Walker RJ. Glycated albumin is the preferred marker for assessing glycaemic control in advanced chronic kidney disease. NDT Plus 2011;4:368-75.

$38 \mathrm{Ng} \mathrm{JM}$, Jennings PE, Laboi $\mathrm{P}$, et al. Erythropoetin treatment significantly alters measured glycated haemoglobin ( $\mathrm{HbA1c})$. Diabet Med 2008;25:239-40.

39 Vos FE, Schollum JB, Coulter CV, et al. Red blood cell survival in patients on long-term dialysis. Am J Kidney Dis 2011;58:591-8.

$40 \mathrm{Kim} \mathrm{C}$, Bullard KM, Herman WH, et al. Association between iron deficiency and A1c levels among adults without diabetes in the National Health and Nutrition Examination Survey, 1999-2006. Diabetes Care 2010;33:780-5.

41 Gan T, Liu X, Xu G. Glycated albumin versus HbA1c in the evaluation of glycemic control in patients with diabetes and CKD. Kidney Int Rep 2018;3:542-54. 